\title{
Réactivation de gènes et mutants de méthylation chez la souris
}

Le rôle de la méthylation de l'ADN chez les eucaryotes apparaît très important mais ses modalités restent discutées. La présence de 5-méthyl-cytosine pourrait affecter l'activité transcriptionnelle en modulant les interactions ADN-protéines. Une difficulté est que la mesure globale de méthylation d'un ADN ou même d'un gène ne reflète qu'imparfaitement les détails cruciaux de la réaction. Un système d'étude très efficace est celui des cellules CHO (chinese hamster ovaries). Une équipe anglaise [1] a utilisé des cellules $\mathrm{CHO}$ négatives à la fois pour la thymidine kinase et pour la métallothionéine $\left(\mathrm{TK}^{-} \mathrm{MT}^{-}\right)$. Deux méthodes de déméthylation ont été employées. La première, déjà classique, consiste à utiliser la 5-azacytidine; on peut ainsi obtenir environ $1 \%$ de révertants $\mathrm{TK}^{+}$parmi les cellules survivantes. Cette proportion monte à $38 \%$ si l'on emploie la 5-désoxyazacytidine, et peut s'élever à près de $60 \%$ si on recherche les $\mathrm{MT}^{+}$. La deuxième méthode consiste à obtenir, à partir de cellules $\mathrm{TK}^{-}$ $\mathrm{MT}^{2}$, des révertants thermosensibles qui soient à la fois $\mathrm{TK}^{+}$et $\mathrm{MT}^{+}$à $39^{\circ} \mathrm{C}$, alors qu'à $34^{\circ} \mathrm{C}$ la réversion n'est pas obtenue (variant $\mathrm{tsm}$, temperature-sensitive methylation). La lignée tsm a été maintenue sur plus de 100 générations.

L'analyse de la méthylation après 5-azacytidine montre une réduction globale d'environ $45 \%$ alors qu'aucune baisse n'est décelable sur les tsm. Mais le point intéressant est donné par l'analyse du gène TK lui-même. Une région particulière, voisine du troisième
$\mathrm{TK}^{+}$, quel que soit son mécanisme de production. En revanche, la région du promoteur en '5' n'est pas altérée. Un phénomène inattendu est que les révertants $\mathrm{TK}^{+}$voient leur degré de méthylation globale augmenter progressivement, de sorte qu'après 100 générations elle est devenue supérieure à celle des témoins $\mathrm{TK}^{-}$, mais la différence au voisinage de l'exon 3 persiste.

Les conclusions que proposent les auteurs vont plus loin que ne le suggère le simple examen de leurs expériences. Ils pensent avoir isolé une souche présentant une lésion de la voie de méthylation de l'ADN à un stade donné. Ce devrait être le chef de file de toute une série d'autres mutants, déficients à différents stades de cette voie. On pourrait ainsi suivre à la fois la régulation et le rôle biologique de la méthylation de l'ADN. Les anomalies de la méthylation retentiraient sur plusieurs gènes et conduiraient à une symptomatologie pléiotrope avec défauts multiples d'expression. De telles maladies ne seraient pas nécessairement héréditaires mais pourraient provoquer des malformations de gravité variable, notamment chez le foetus.

J.-C. D.

1. Gounari F, Banks GR, Khazaie K, Jeggo PA, Holliday R. Gene reactivation : a tool for the isolation of mammalian DNA methylation mutants. Genes Dev $1987 ; 1: 699-912$.
Nos ancêtres mangeaient du soufre et vivaient dans l'eau bouillante! Une récente réévaluation des arbres évolutifs des êtres vivants basée sur l'évolution des ARN ribosomaux a été présentée par J.A. Lake [1]. Elle confirme ses travaux antérieurs, à savoir que le dernier ancêtre commun aux eucaryotes et aux procaryotes (pour utiliser une classification usuelle rendant compte peut-être mal des données actuelles) était proche des éocytes [1, 2], c'est-àdire des archebactéries dépourvues de noyau, métabolisant les dérivés soufrés et vivant à des températures très élevées. Les autres archebactéries et les eubactéries (c'est-à-dire les bactéries actuelles) dériveraient d'une branche différente de celle des éocytes et des eucaryotes.

[1. Lake JA. Nature 1988, 331 : 184-6.]

[2. Cocito C. médecine/sciences 1986 ; 2 : 436-44.]

- Quand un facteur de croissance sort de son trou... gare! Le FGF basique (fibroblast growth factor) est un puissant facteur de croissance, abondant dans des cellules qui ne prolifèrent pas: cerveau, cartilage, rien. En fait, la protéine normale est dépourvue de «peptide signal» $\left(m / s n^{\circ} 6\right.$, vol. 2, p. 341) et reste donc intracellulaire en dehors des phénomènes d'attrition tissulaire. Cependant, lorsqu'un peptide signal est ajouté à cette protéine par recombinaison génétique, les cellules produisant cette protéine modifiée prolifèrent et sont tumorigènes alors que des cellules produisant une quantité comparable de FGF dépouvu de peptide signal ont un phénotype normal. Ces résultats démontrent que le FGF est un oncogène potentiel dont l'action nécessite une sécrétion lui permettant, au sein d'une boucle autocrine, de se fixer à ses récepteurs membranaires et de stimuler la prolifération.

[Rogelj S, et al. Nature 1988, 331 : 173-5.] 


\section{BRÈVES}

Les neurophysiologistes qui étudient les circuits de la mémorisation viennent de marquer un point en découvrant une population de neurones qui gardent, pendant 16 secondes au moins, la trace d'une image aux formes précises [1]. On avait déjà décrit, dans le lobe temporal des primates, des neurones répondant spécifiquement à la présentation d'une image aux formes précises par une activité phasique (durant la présentation de l'image). Pour étudier la mémorisation, il ne suffit cependant pas d'enregistrer une réponse phasique, mais une réponse soutenue après retrait de la stimulation visuelle. Le singe est, pour cela, mis en situation de tâche avec délai : on lui présente une image très brièvement $(0,2 \mathrm{~s})$, puis si, et seulement si cette image a les caractéristiques qu'on lui a appris à reconnaître, il doit appuyer sur une manette 16 secondes plus tard lorsqu'on allume une lampe-test. On avait décrit, ainsi, des neurones qui gardaient la trace de la présentation d'une couleur dans le champ visuel, c'est-à-dire qui présentaient une activité soutenue après présentation de la couleur durant les 16 secondes de délai. Une équipe japonaise [1] vient donc de découvrir des neurones gardant la trace non plus seulement d'une couleur mais bien d'une image aux formes précises. Il s'agit d'une nouvelle pierre dans la reconstruction des circuits de la mémoire. Mais il ne s'agit semblet-il pas que de cela, car Miyashita et Chang tirent des conclusions conceptuelles fondamentales d'une de leurs observations: les neurones à mémoire de formes sont en fait des neurones répondant par ailleurs d'une façon bien connue à des stimulations visuelles particulières. La mémorisation de formes (à court terme) serait ainsi liée à l'activation temporaire de neurones impliqués par ailleurs dans d'autres tâches et non pas à l'activation d'une population spécifique de $\mathrm{m} / \mathrm{s} n^{\circ} 4$ vol. 4, avril 88
« neurones mnémoniques » situés dans une zone corticale spécifique. La mémorisation comme activité secondaire des aires corticales sensorielles associatives, voilà une hypothèse à suivre !

[1. Miyashita Y, Chang HS. Nature 331 ; 1988 : 68-70.]

Le gène $\delta$ du récepteur pour l'antigène des lymphocytes $T$ a une partie variable constituée de trois ou quatre segments génétiques. Il existe deux types de récepteurs pour l'antigène des lymphocytes $T$. Le plus commun, et le plus anciennement connu, est constitué des chaînes $\alpha$ et $\beta$ [1]. Plus récemment, un second type de récepteur, constitué des sousunités $\gamma$ et $\delta$, a été décrit au niveau de populations minoritaires de cellules $\mathrm{T}$ et de lymphocytes à un stade très précoce de leur différenciation ([2] et $\mathrm{m} / \mathrm{s} n^{\circ} 7$, vol. 3, p. 431). Les gènes codant pour toutes ces chaînes subissent, au cours de la différenciation, un réarrangement spécifique aboutissant aux gènes fonctionnels. Les parties variables sont constituées par la juxtaposition de segments $V$ et $J$ (pour les gènes $\alpha$ et $\gamma \ldots$ comme pour les gènes des chaînes légères d'immunoglobuline)... ou $\mathrm{V}, \mathrm{D}$ et $\mathrm{J}$ (pour le gène $\beta$... comme pour celui des chaînes lourdes d'immunoglobuline). C'est encore un processus plus compliqué qui aboutit à la constitution de la séquence codant pour la partie variable de la chaîne $\delta$ : ici, jusqu'à quatre segments génétiques sont impliqués $\left(\mathrm{V}, \mathrm{D}_{1}, \mathrm{D}_{2}\right.$, et $\left.\mathrm{J}\right)$ [3]. Par ailleurs, les résultats récents confirment que le locus $\delta$ est entièrement inclus dans le locus $\alpha$, comme nous l'avions indiqué précédemment $\left(m / s n^{\circ} 7\right.$, vol. 3 , p. 431).

[1. Malissen M, Malissen B. médecine/sciences 1986; 2 : 30411.]

[2. Lefranc MP. médecine/ sciences $1987 ; 3: 150-6$.]

[3. Chien YH. Nature 1987; 330 : 722-7.]
La prolactine est-elle une hormone immunopermissive? Chez la souris, la bromocryptine, un agoniste du récepteur de la dopamine qui inhibe la sécrétion de prolactine, provoque une immunosuppression importante. $\mathrm{La}$ réponse à une infection par Listeria monocytogenes, la sécrétion de $\gamma$-interféron par les lymphocytes $T$ et la stimulation par les mitogènes de la prolifération de lymphocytes spléniques sont diminuées par la bromocryptine et normalisées par l'injection de prolactine. La survie des animaux infectés par des Listeria est également diminuée par la bromocryptine et normalisée par la prolactine. Chez la souris, cette hormone semble donc exercer un rôle important sur la fonction des lymphocytes $\mathrm{T}$. Quelques arguments cliniques laissent supposer que chez l'homme aussi les inhibiteurs de la sécrétion de prolactine pourraient avoir des effets immunosuppresseurs.

[Bernton EW, et al. Science 1988 : $239: 401-4$.]

Les G-protéines sont-elles des oncogènes potentiels? Tout récepteur hormonal, toute G-protéine et toute enzyme « effectrice » contrôlant la concentration de seconds messagers, sont des «oncogènes potentiels » pour des cellules dont la croissance est stimulée par la mise en jeu de ces systèmes. Ainsi existe-t-il une sous-classe de tumeurs hypophysaires sécrétrices d'hormone de croissance dont la synthèse d'AMP cyclique, normalement stimulée par la libérine hypothalamique spécifique (GHRH, growth hormone releasing hormone), est constitutivement forte. L'anomalie moléculaire semble être ici une activation de la sousunité $\alpha$ de la $\mathrm{G}$-protéine $\mathrm{G}_{\mathrm{S}}$, anomalie dont il n'est pas établi si elle est primitive (mutation ponctuelle) ou secondaire (activation par un mécanisme semblable à 'ADP-ribosylation catalysée par la toxine cholérique).

[Vallar L, et al. Nature 1987; 330 : 566-8. ] 
Des canaux ioniques dans la membrane interne des mitochondries. Le rôle principal des mitochondries est de produire de l'énergie grâce aux réactions d'oxydation des coenzymes réduits, réactions couplées au passage de protons dans l'espace intermembranaire, c'est-à-dire à la face externe de la membrane interne. Le gradient de $\mathrm{pH}$ et le gradient osmotique ainsi créés entre la face externe et la face interne de la membrane interne sont utilisés pour fournir l'énergie nécessaire à la phosphorylation d'ADP en ATP, principale molécule de stockage de l'énergie chez les êtres vivants. L'utilisation de la technique du patch clamp à l'étude de la membrane mitochondriale a permis à une équipe italo-allemande de démontrer l'existence de canaux voltagedépendants spécifiques des anions a u niveau de la membrane mitochondriale interne [1]. L'ouverture de ces canaux, permettant le passage d'anions $\mathrm{OH}^{-}$dans l'espace intermembranaire et donc la production d' $\mathrm{H}_{2} \mathrm{O}$ et la disparition du gradient de protons, crée un découplage entre les réactions d'oxydation et la synthèse d'ATP, l'énergie des réactions chimiques étant alors dissipée sous forme de chaleur [2]. C'est un mécanisme de ce type qui est impliqué dans la production de chaleur par la graisse brune [3], la protéine constituant le canal à anion étant alors la thermogénine.

[1. Sorgato MC, et al. Nature 1987; 330: 498-500.]

[2. Selwyn MJ. Nature $1987 ; 330$ : 424-5.]

[3. Ricquier D. médecine/sciences $1985 ; 1$ : 147-53.]

- Un traitement de la maladie de Huntington expérimentale. La maladie de Huntington, une des plus redoutables affections du système nerveux, peut être simulée chez le rat par l'administration d'acide quinolinique $\left(\mathrm{m} / \mathrm{s} n^{\circ} 7\right.$, vol. 2, p. 400). Cette «excitotoxine » agit en activant un récep- parce qu'il est activé par l'acide $\mathrm{N}$-méthyl $\mathrm{D}$ aspartique, analogue synthétique des excitateurs naturels que sont les acides glutamique et aspartique. Cet agent ouvre un canal calcique, et c'est le calcium en excès qui attaque les cellules, notamment les neurones producteurs de l'inhibiteur GABA (acide $\gamma$ aminobutyrique). Parmi les produits essayés pour bloquer la toxicité de l'acide quinolinique chez le rat, un s'est montré efficace. Ce «MK-801 » se fixe au récepteur en neutralisant complètement les effets de l'acide quinolinique; de plus, sa valeur thérapeutique éventuelle tient à sa liposolubilitéqui lui permet de traverser la barrière hémo-méningée et donc d'être utilisable par voie orale. On est encore sans doute loin du traitement de la maladie de Huntington; mais le rythme des progrès nous paraît suffisant pour pouvoir d'ores et déjà pallier une des situations les plus pénibles de la «médecine prédictive » : la connaissance précise du locus de la maladie sur le chromosome $4\left(\mathrm{~m} / \mathrm{s} n^{\circ} 2\right.$, vol. 4 , p. 128) permettra de prévoir, dans une famille, qui est destiné à être atteint et qui ne l'est pas; si l'échéance, pour un sujet jeune, se situe dans 20 ou 30 ans, on peut raisonnablement lui faire espérer d'ici-là la découverte d'un traitement actif, curatif ou préventif.

[Marx JL. Science 1987; 238: 1510-1.]

- La normalisation glycémique obtenue par greffe de pancréas chez des diabétiques insulinodépendants ne ralentit pas l'évolution de la rétinopathie diabétique. L'évolution d'une rétinopathie diabétique préexistante à la greffe a été appréciée chez 27 malades ayant subi une greffe de pancréas à Minneapolis (Minnesota, USA), greffe fonctionnelle depuis plus d'une année. Chez tous ces malades, le contrôle de la glycémie a été normalisé. Aucune différence n'a cependant été constatée dans l'évolution de la rétinopathie entre ces diabétiques greffés, normoglycémiques, et des diabétiques plus ou moins bien équilibrés par un traitement insulinique conventionnel. Dans certains cas, au contraire, le contrôle étroit du diabète semble avoir, durant les premiers mois, aggravé les lésions rétiniennes, peut-être du fait de la réduction du débit sanguin rétinien qu'entraîne la disparition de l'hyperglycémie. Ces résultats décevants ne signifient cependant pas qu'une greffe précédant toute lésion rétinienne ne serait pas de nature à empêcher l'apparition de la rétinopathie diabétique.

[Ramsay RC, et al. N Engl J Med 1988 ; $318: 208-14$.]

- Un gène impliqué dans l'invasivité et le pouvoir métastasiant des tumeurs est-il situé sur le chromosome 7 humain ? Lorsque des cellules d'un lymphome de souris non invasif et non métastasiant sont fusionnées avec des cellules humaines, l'hybride somatique ainsi obtenu est hautement tumorigène chez la souris, provoquant des métastases. La culture prolongée de tels hybrides somatiques homme/rongeur est associée à la perte progressive et apparemment au hasard de chromosomes humains, ce qui permet de corréler une caractéristique phénotypique à un chromosome humain particulier. Ainsi peuton observer que le pouvoir invasif des hybrides décrits ci-dessus est perdu parallèlement à la perte du chromosome 7 , qui pourrait donc contenir un ou plusieurs gènes intervenant dans le potentiel prolifératif et la diffusion des cellules l y m p om a te u ses mu rines. On sait que le chromosome 7 contient deux oncogènes potentiels, $c$-erb $B$ et le gène codant pour la chaîne A de PDGF (platelet-derived growth factor), l'intervention de ces deux gènes dans la stimulation de la tumorigénicité des hybrides reste cependant hypothétique.

[Collard JG, et al. Cancer Res 1987 ; 47 : 6666-70.] 